\title{
Transcriptional diversity of DMRT1 (dsx- and mab3-related transcription factor 1) in human testis
}

\author{
Han Hua Cheng ${ }^{1}$, Ming Ying ${ }^{1}$, Yi Hao Tian ${ }^{1}$, Yiqing Guo ${ }^{1}$, Ken McElreavey ${ }^{2}$, Rong Jia Zhou ${ }^{1}$ \\ ${ }^{1}$ Department of Genetics and Center for Developmental Biology, College of Life Sciences, Wuhan University, Wuhan 430072, China; \\ ${ }^{2}$ Department of Developmental Biology, Institut Pasteur, 25 rue du Dr Roux, 75724 Paris Cedex 15, France
}

Recent advances in the evolutionary genetics of sex determination indicate that the only molecular similarity in sex determination found so far among phyla is between the fly doublesex, worm mab-3 and vertebrate DMRT1(dsx- and mab3-related transcription factor 1) /DMY genes. Each of these factors encodes a zinc-finger-like DNA-binding motif, DM domain. Insights into the evolution and functions of human DMRT1 gene could reveal evolutionary mechanisms of sexual development. Here we report the identification and characterization of multiple isoforms of human DMRT1 in the testis. These transcripts encode predicted proteins with 373, 275 and 175 amino acids and they were generated by alternative splicing at $3^{\prime}$ region. Expression level of DMRT1a is higher than those of both DMRT1b and $c$, and the $D M R T 1 c$ expression was the lowest in testis, based on comparisons of mean values from real-time fluorescent quantitative RT-PCR analysis. Both DMRT1b and $c$ result from exonization of intronic sequences, including the exonization of an Alu element. A further search for Alu elements within the DMRT1 gene demonstrated that all 99 Alu elements are non-randomly distributed among the non-coding regions on both directions. These new characteristics of $D M R T 1$ would have an important impact on the evolution of sexual development mechanisms.

Cell Research (2006) 16:389-393. doi:10.1038/sj.cr.7310050; published online 13 April 2006

Keywords: evolution, alternative splicing, doublesex, sex differentiation

\section{Introduction}

In mammals, male sex determination starts by activation of the testis-determining factor gene, $S R Y$ (sex-determining region on $Y$ chromosome) [1]. This gene encodes an HMG domain transcription factor that triggers the differentiation of Sertoli cells, and therefore testis formation. Although several genes involved in gonadal differentiation have been identified including $S O X 9$ (an $S R Y$-related gene that contains a similar HMG box domain), DAXI (an X-chromosome-located orphan nuclear receptor), $S F 1$ (steroidogenic factor, another orphan nuclear receptor) and WT1 (Wilms tumor suppressor), many genes involved in sex determination and differentiation genes are unidentified.

Correspondence: Rong Jia Zhou

Fax: 86-27-68756253;

E-mail: rjzhou@whu.edu.cn

Received 1 Sep 2005; revised 20 Dec 2005; accepted 23 Jan 2006, published online 13 Apr 2006
A wild-type $S R Y$ gene has been observed in over $80 \%$ of patients with 46,XY pure gonadal dysgenesis, suggesting that other genes are involved [2]. A gene termed DMRT1 ( $\underline{d} s x$ - and $\underline{m} a b 3$-related transcription factor 1$)$ is located at the distal portion of chromosome $9 p$ and this gene is deleted in the monosomy $9 \mathrm{p}$ syndrome that includes defective testis development and occasional male-to-female sex reversal [3-5]. The human DMRT1 gene shows significant molecular similarity to doublesex ( $d s x$ ) (Drosophila) and mab-3 (Caenorhabditis elegans) [6-8]. These genes encode putative transcription factors that have a common DNAbinding domain, termed the DM domain. Both $d s x$ and mab-3 control aspects of sex-specific differentiation and are functionally related [7]. Male-specific expression for the $D M R T 1$ gene in early gonadogenesis is consistent with its role in human testis development [9]. A Dmrtl knockout mouse also demonstrated that the gene is required for testis differentiation after determination, but dispensable for ovary development [10]. Furthermore, the Dmrt1 gene transposed to the $\mathrm{Y}$ chromosome in some fish (medaka) has 
become a master regulator gene (Dmrt1y/DMY) in male determination $[11,12]$. In birds, the Dmrt1 is sex-linked [13] on the $\mathrm{Z}$ chromosome, and there is a higher dosage of the gene in the male (ZZ) as compared with the female (ZW) $[8,14-16]$. These data show that the expression of the DM genes from invertebrates to vertebrates is sex-specific (or sex differential) and (with the exception of Drosophila) associated with male-specific development. However, we know remarkably little about the evolution and functions of the DM genes.

Here we identify and characterize multiple isoforms of human DMRT1 that were spliced alternatively in the testis. These transcripts contain coding regions by exonization of intronic sequences, especially by the exonization of Alu elements with the DMRT1 gene. The alternatively spliced Alu exons (AExs) enrich the gonad transcriptome and enhance the coding capacity, suggesting that Alu exonization is an evolutionary pathway that creates human-specific transcriptomic diversity.

\section{Materials and methods}

\section{RACE analysis and cloning of alternatively spliced cDNAs} of DMRT1

On the basis of the sequence information of the DM domain of human DMRT1, RACE primers were designed. 5' RACE was performed using human testis cDNAs (Marathon ${ }^{\mathrm{TM}} \mathrm{cDNA}$, CLONTECH Laboratories) as template. The primers were AP2, 5' ACT CAC TAT AGG GCT CGA GCG GC 3' and DM primer 3-1, 5'ACA AGC AGC TCG GCC GCA CTG 3'. We performed 3' RACE using primers AP1 and DM primer 5-1, 5' TGG GTG CCG GGA GCA AGA AGT3'. After the PCR, nest PCR was carried out using primers AP2, and DM primer 5-N, 5' TTT GCG GCC GCA AGT GCG CAC GCT GCA GGA AC3'. RACE cycling conditions were as follows: 35 cycles, each with $30 \mathrm{~s}, 94^{\circ} \mathrm{C} ; 2 \mathrm{~min}, 70{ }^{\circ} \mathrm{C}$ in a $20 \mathrm{ml}$ Advantage 2 Polymerase Mix (CLONTECH Laboratories). All bands amplified were gel-purified, cloned into the pCRII (Invitrogen, USA) and sequenced using an $\mathrm{ABI} 377$ autosequencer.

\section{RNA isolation, $c D N A$ synthesis and RT-PCR}

All the RNAs were prepared by the RNeasy Mini kit (Qiagen, USA) according to the manufacturer's instructions, digested by Rnase-free DNase I and purified. About $3 \mu \mathrm{g}$ RNAs were used as template for reverse transcription using $0.5 \mu \mathrm{g}$ poly $(\mathrm{T})_{20}$ primer and 200 U MMLV reverse transcriptase (Promega, USA). RT-PCR (non quantification) was used to amplify individual isoforms of DMRT1 from the adult testis cDNA. PCR cycling condition were as follows: 35 cycles, each with $30 \mathrm{~s}, 94{ }^{\circ} \mathrm{C} ; 30 \mathrm{~s}, 68{ }^{\circ} \mathrm{C} ; 30 \mathrm{~s}, 72{ }^{\circ} \mathrm{C}$ in a $20 \mu 1$ reaction mix containing $10 \mathrm{mM}$ Tris- $\mathrm{HCl} \mathrm{pH} 8.3,1.5 \mathrm{mM} \mathrm{MgCl}_{2}, 50$ $\mathrm{mM} \mathrm{KCl}, 200 \mu \mathrm{M}$ dNTP, $0.2 \mu \mathrm{M}$ each primer, $1 \mathrm{U}$ Taq DNA polymerase. The primers were as follows: sense primer for DMRT1 $a, b$ and $c$, 5' CGG CTG CCC AAG TGC GCA 3', and antisense primers, 5' CTC GTC CTC CTC GAT GAC GG 3' for DMRT1a; 5' CAA GTG CGT GTG TGC CTC TG 3' for Dmrt1b, and 5' CTA CTT GGG AGG CTG AGG CAG 3' for Dmrtlc, and antisense nest primer 5' GCG CTT GTG GCC CTT GAG CG 3' for Dmrtlc, and GAPD primers:
5' TCC AAA ATC AAG TGG GGC GA $3^{\prime}$ and 5' AGT AGA GGC AGG GAT GAT GT 3'.

\section{Real time fluorescent quantitative RT-PCR}

Real time RT-PCR was used for the quantification of the expression of both DMRTl $a$ and $b$ using the multi-channel RotorGene 3000 (Corbett Research, Australia), according to the supplied protocol. PCR cycling condition were as follows: 5 min at $95{ }^{\circ} \mathrm{C}$; 40 cycles of $30 \mathrm{~s}$ at $95{ }^{\circ} \mathrm{C}, 30 \mathrm{~s}$ at $68{ }^{\circ} \mathrm{C}, 30 \mathrm{~s}$ at $72{ }^{\circ} \mathrm{C}$ in a $25 \mu 1$ reaction mix containing $0.5 \times$ Sybr Green I. The primers used were the same as those in RT-PCR. A serial dilution of cDNA samples was simultaneously used to amplify GAPD to determine the quantity of cDNAs as standards. Based on the standards, the DMRT1 cDNAs were then amplified using a serial dilution of the cDNAs as templates, and the relative expression levels of the $D M R T 1$ to $G A P D$ were determined. For robustness issues, each sample was performed in triplicate or more. Data were analyzed by the software Rotor-gene version 4.6.

\section{DMRT1 gene structure analysis}

All sequences were analyzed with the Vector NTI software. Sequence alignments were performed using the ClustalW program. Genomic structure of the DMRT1 was analyzed by searching GenBank, NCBI online, and by comparison of cDNAs with genomic contig, which encodes the DMRT1 cDNA.

\section{Alu element analysis}

The Alu element similarity searches were carried out by BLAST online. All Alu elements within the genomic sequence of $D M R T 1$ were counted and their directions on both strands were determined.

\section{Results}

\section{Alternative splicing of the DMRT1}

In an attempt to isolate the DMRT1 transcripts from the human testis to determine transcriptional diversity, the Marathon cDNAs of the human testis synthesized from the AP primer were used as template for both 5' RACE and 3' RACE by combined use of the primer pairs of DM domain and AP primer and nested PCR. RACE PCR results indicated several bands in 3' RACE, while a specific band was observed for the 5' RACE. Sequencing analysis demonstrated that there are three transcripts (Figure 1). Interestingly, sequencing comparisons indicated two kinds of alternative splicing events of the DMRT1 transcript in the human testis, all of which occurred in the region 3' to the DM domain. Three spliced transcripts, DMRT1a, $D M R T 1 b$ and DMRT1c, were identified in the testis and they encoded three predicted proteins of 373,275 and 175 aa, respectively (Figure 2). The expression patterns of these spliced isoforms were analyzed by RT-PCR and quantitated using real-time fluorescent quantitative RTPCR from adult testis. Expression level of DMRTIa is the highest among these transcripts and 4.4 times higher than DMRT1b expression (Figure 3A and B). DMRT1c expressed much lower and can only be detected by nest 


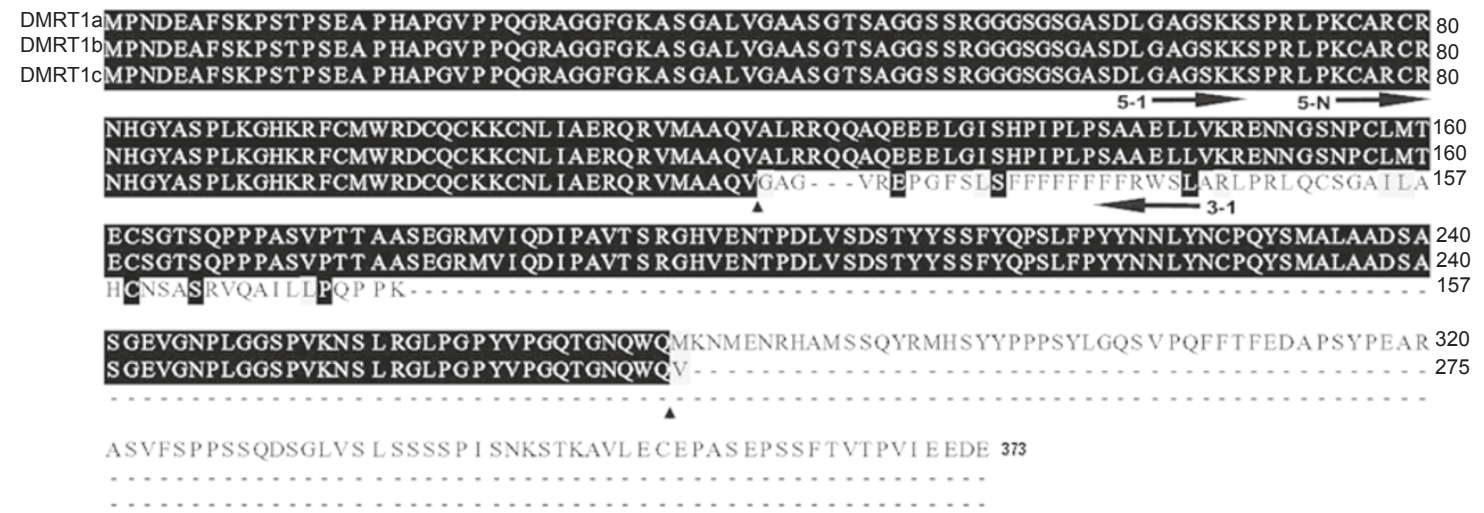

Figure 1 Alignment of predicted amino-acid sequences of three isoforms of human DMRT1; GenBank accession numbers are AY442914 for DMRT1b and AY442915 for DMRT1c. Triangles indicate the splicing sites. Arrows show the primer positions for RACE analysis.

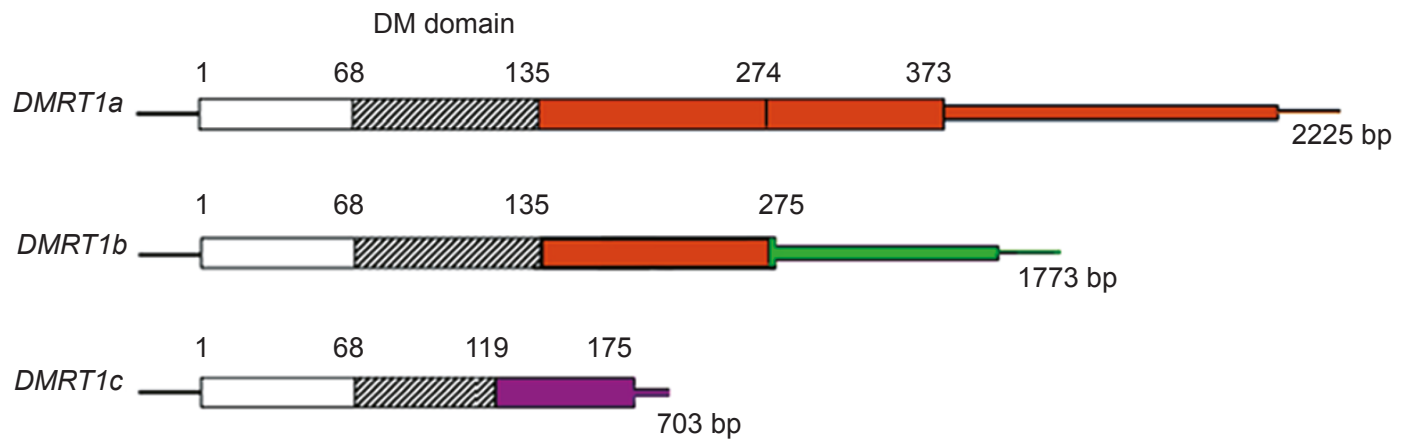

Figure 2 Diagram of alternatively spliced transcripts of human DMRT1 gene. DMRT1 is spliced to form different mRNAs transcripts: DMRT1a, DMRT1b and DMRT1c, which encode DM proteins with different amino acids (numbers above each line). DM domains are indicated by shaded boxes. Alternatively spliced regions in 3' region are showed by different color. In Dmrt $1 c$, the splicing event occurred within the DM domain. Therefore, the new transcript missed the distal part of the DM domain. The numbers in the end under the lines indicate nucleotide numbers of these cDNAs.

PCR (Figure 3A).

Gene structure and exonization of intronic sequences of human DMRT1

After comparing the genomic sequence with mRNA sequences, we found that there were seven exons, which were alternatively spliced to generate multiple DMRT1 isoforms (Figure 4). In isoform DMRT1b, exon 3 continues to read through intron 3 to form a new exon 6, while in DMRT1c, exon 1 continues to read through intron 1 to form another new exon 7 , suggesting that alternative splicing enriches the gonad transcriptome and enhances the coding capacity.

\section{Exonization of Alu elements of the human DMRT1}

A BLAST search against these isoforms indicated that half of the DMRT1c exon 7 was generated by the exonization of an Alu sequence in intron 1. A further screen for
$A l u$ elements within the whole gene revealed that $99 \mathrm{Alu}$ elements in the DMRT1 gene are distributed among the non-coding regions in both directions, especially within introns, except for the exon 7 of the DMRT1c (Figure 4), which suggested that the locations of the Alu elements within the DMRT1 are not random.

\section{Discussion}

The surprisingly low number of genes in the human genome suggested that alternative splicing could account for biological complexity. Indeed, bioinformatic analyses indicate that at least $50 \%$ of all human genes participate in alternative splicing, which contributes significantly to human proteome complexity $[17,18]$. Alternative splicing is often regulated according to tissue type, developmental stage, sex or physiological conditions. Aberrant regulation 

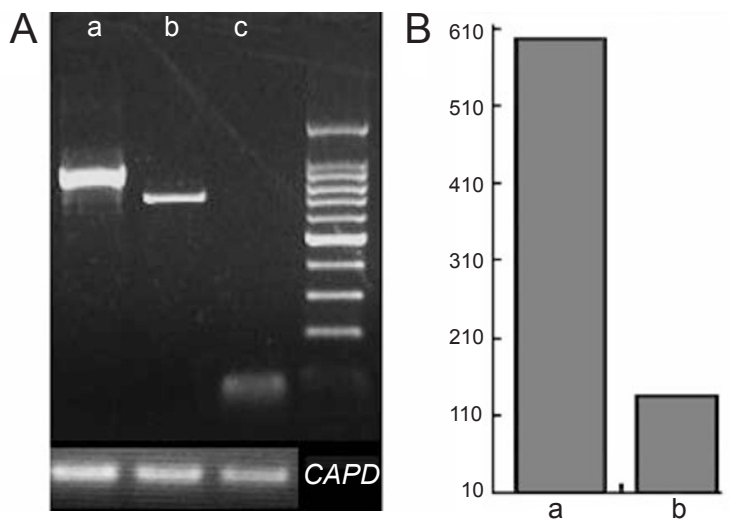

Figure 3 Non-quantitative RT-PCR (A) and Real time fluorescent quantitative RT-PCR (B) analysis of DMDRT1. (A) Non-quantitative RT-PCR of DMRT1 transcripts shows their expression in adult testis. Both DMRT1 a and b can be amplified by first PCR; however, $D M R T 1 c$ showed much lesser expression and can only be detected by nest PCR. PCR amplification of GAPD was used as a control. 100 bp DNA ladder was loaded on the right lane as a molecular weight marker. Real-time fluorescent quantitative RT-PCR analysis (B) shows the relative expression of both DMRTI $a$ and $b$ to GAPD in the testis. Data were analyzed by the Rotor-gene software, version 4.6.

of alternative splicing has been implicated in an increasing number of human diseases, including cancer [19]. Human DMRT1 in testis tends to be spliced alternatively at $3^{\prime}$ region. Interestingly, alternative splicing events at 3' termini of several DM-domain genes were also observed: for example, $d s x$ of Drosophila [20] and zebrafish Dmrt1
[21]. The $d s x$ is alternatively spliced at 3 ' region, by default, into the male form $d s x^{m}$, and the female $d s x^{f}$ is generated by a female-specific TRA. Diverse isoforms of the DM genes generated by alternative splicing at $3^{\prime}$ termini may provide potentially diverse targets for different upstream and downstream interacting factors in sexual regulation. Few targets of regulation by DM factors have yet been clearly identified, and most of the data are obtained from $d s x$ of Drosophila and $m a b-3$ of C. elegans [22, 23]. Further identification of interactive factors with $D M R T 1$ in vertebrates will determine how these spliced DMRT1s operate.

Exonization of intronic sequences is a mechanism that not only enriches the transcriptome and enhances the coding capacity of human genome for a diversity of biological activities [24, 25], but also provides genetic sources for evolution. Exonization of intronic sequences of human DMRT1 was generated by alternative splicing. It seems that alternative splicing might be a force for both enhancement of the coding capacity of human genome and molecular evolution, and DMRT1 as a target of these processes would have an important impact on the evolution of sexual development mechanisms. Alu sequences are short interspersed elements (SINEs), typically 300 nucleotides in length. Alu elements multiply within the genome through RNA polymerase III-derived transcripts in a process termed retroposition. Despite their lack of known function, recent findings suggests that $A l u$ elements have a broad evolutionary impact [26]. In human DMRT1, non random distribution of Alu elements on both directions of both DNA strands suggest a biological role. Alu-mediated homologous unequal recombination may result in genetic

Clone RP11-143M15

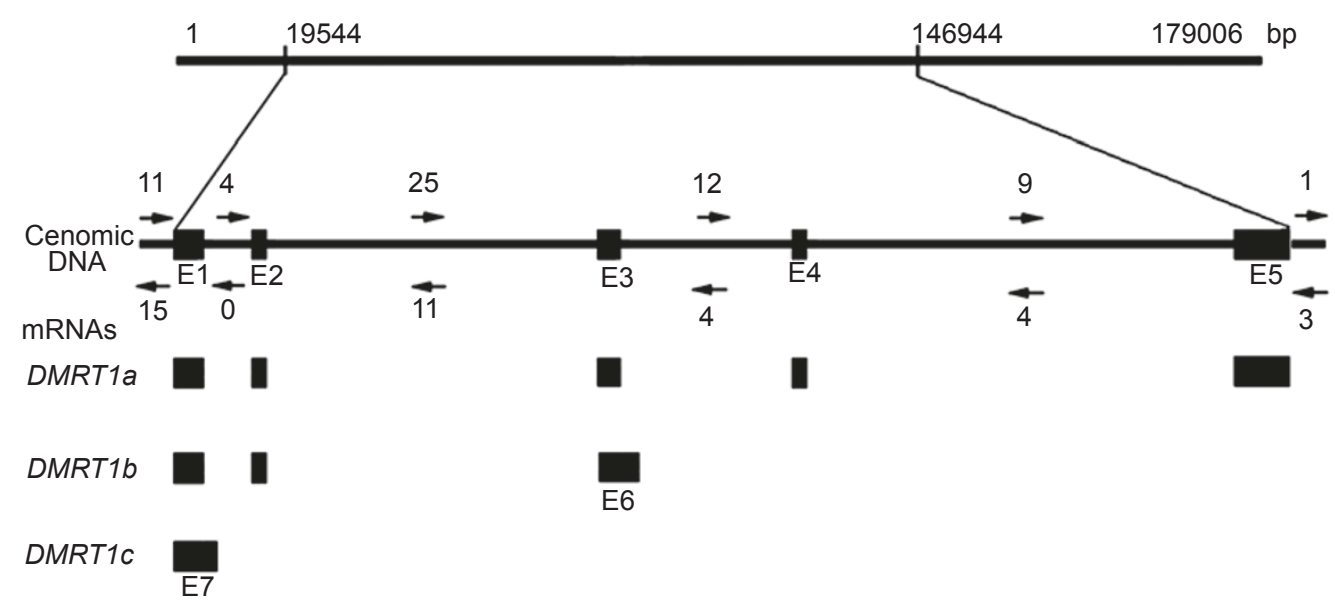

Figure 4 Schematic representation of the genomic organization and mRNA sequences of human DMRT1. Lines at the top show the genomic contig from gene database. Solid bars represent exons (E1-E7), whereas introns are indicated by the straight lines. Non random distribution of $A l u$ elements on both directions of both DNA strands of human DMRT1 gene. Arrows and numbers indicate numbers and locations of $A l u$ elements on sense and antisense strand, respectively. Arrows indicate the directions of $A l u$ elements. 
defects. Exonization of Alu elements, that is, insertion into mature mRNAs by alternative splicing, generated isoforms of DMRT1, which might provide a redundant/alternative way for functions of the gene in spermatogenesis, as sexual development is an essential process of reproduction.

\section{Acknowledgments}

The work was supported by the National Natural Science Foundation of China, and the National Key Basic Research project (2004CB117400), the Program for New Century Excellent Talents in University and the Key Project of Chinese Ministry of Education (No. 2004.28).

\section{Ref erences}

1 Swain A, Lovell-Badge R. Mammalian sex determination: a molecular drama. Genes Dev 1999; 13:755-767.

2 McElreavey K, Fellous M. Sex determination and the Y chromosome. Am J Med Genet 1999; 89:176-185.

3 Guioli S, Schmitt K, Critcher R, et al. Molecular analysis of 9p deletions associated with XY sex reversal: refining the localization of a sex-determining gene to the tip of the chromosome. Am J Hum Genet 1998; 63:905-908.

4 Ion R, Telvi L, Chaussain JL, et al. Failure of testicular development associated with a rearrangement of $9 \mathrm{p} 24.1$ proximal to the SNF2 gene. Hum Genet 1998; 102:151-156.

5 Ounap K, Uibo O, Zordania R, et al. Three patients with 9p deletions including DMRT1 and DMRT2: a girl with XY complement, bilateral ovotestes, and extreme growth retardation, and two XX females with normal pubertal development. Am J Med Genet A 2004; 130A:415-423.

6 Raymond CS, Parker ED, Kettlewell JR, et al. A region of human chromosome $9 p$ required for testis development contains two genes related to known sexual regulators. Hum Mol Genet 1999; 8:989-996.

7 Raymond CS, Shamu CE, Shen MM, et al. Evidence for evolutionary conservation of sex-determining genes. Nature 1998; 391:691-695.

8 Raymond CS, Kettlewell JR, Hirsch B, et al. Expression of Dmrt1 in the genital ridge of mouse and chicken embryos suggests a role in vertebrate sexual development. Dev Biol 1999; 215:208220.

9 Moniot B, Berta P, Scherer G, et al. Male specific expression suggests role of DMRT1 in human sex determination. Mech Dev 2000; 91:323-325.

10 Raymond CS, Murphy MW, O’Sullivan MG, et al. Dmrt1, a gene related to worm and fly sexual regulators, is required for mammalian testis differentiation. Genes Dev 2000; 14:2587-2595.
11 Matsuda M, Nagahama Y, Shinomiya A, et al. DMY is a Yspecific DM-domain gene required for male development in the medaka fish. Nature 2002; 417:559-563.

12 Nanda I, Kondo M, Hornung U, et al. A duplicated copy of DMRT1 in the sex-determining region of the Y chromosome of the medaka, Oryzias latipes. Proc Natl Acad Sci USA 2002; 99:11778-11783.

13 Nanda I, Shan Z, Schartl M, et al. 300 million years of conserved synteny between chicken $\mathrm{Z}$ and human chromosome 9 . Nat Genet 1999; 21:258-259.

14 Smith CA, McClive PJ, Western PS, et al. Conservation of a sex-determining gene. Nature 1999; 402:601-602.

15 Nanda I, Zend-Ajusch E, Shan Z, et al. Conserved synteny between the chicken $\mathrm{Z}$ sex chromosome and human chromosome 9 includes the male regulatory gene DMRT1: a comparative (re)view on avian sex determination. Cytogenet Cell Genet 2000; 89:67-78.

16 Shan Z, Nanda I, Wang Y, et al. Sex-specific expression of an evolutionarily conserved male regulatory gene, DMRT1, in birds. Cytogenet Cell Genet 2000; 89:252-257.

17 Lander ES, Linton LM, Birren B, et al. Initial sequencing and analysis of the human genome. Nature 2001; 409:860-921.

18 Venter JC, Adams MD, Myers EW, et al. The sequence of the human genome. Science 2001; 291:1304-1351.

$19 \mathrm{Xu} \mathrm{Q}$, Lee C. Discovery of novel splice forms and functional analysis of cancer-specific alternative splicing in human expressed sequences. Nucleic Acids Res 2003; 31:5635-5643.

20 Burtis KC, Baker BS. Drosophila doublesex gene controls somatic sexual differentiation by producing alternatively spliced mRNAs encoding related sex-specific polypeptides. Cell 1989; 56:997-1010.

21 Guo Y, Cheng H, Huang X, et al. Gene structure, multiple alternative splicing, and expression in gonads of zebrafish Dmrt1. Biochem Biophy Res Comm 2005; 330:950-957.

22 Yi W, Ross JM, Zarkower D. mab-3 is a direct tra- 1 target gene regulating diverse aspects of $C$. elegans male sexual development and behavior. Development 2000; 127:4469-4480.

23 Yi W, Zarkower D. Similarity of DNA binding and transcriptional regulation by Caenorhabditis elegans mab-3 and Drosophila melanogaster dsx suggests conservation of sex determining mechanisms. Development 1999; 126:873-881.

24 Lev-Maor G, Sorek R, Shomron N, et al. The birth of an alternatively spliced exon: $3^{\prime}$ splice-site selection in Alu exons. Science 2003; 300:1288-1291.

25 Sorek R, Lev-Maor G, Reznik M, et al. Minimal conditions for exonization of intronic sequences: 5 ' splice site formation in alu exons. Mol Cell 2004; 14:221-231.

26 Dagan T, Sorek R, Sharon E, et al. AluGene: a database of Alu elements incorporated within protein-coding genes. Nucleic Acids Res 2004; 32:D489-492.

Edited by Zhong Cheng Zheng 